\title{
TROMBOSIS DE SENO VENOSO POSPARTO: REPORTE DE CASO Y REVISIÓN DE LA LITERATURA*
}

\section{Postpartum cerebral venous sinus thrombosis: a case report and literature review}

Alejandro Hernández-Guzmán, M.D.**, Julián Delgado-Gutiérrez, M.D., MSc.***

Recibido: enero 28/08 - Aceptado: marzo 30/09

\section{RESUMEN}

Presentamos el caso de una paciente joven con síntomas neurológicos: cefalea y convulsiones en el posparto en la cual se sospechó eclampsia posparto o un proceso infeccioso. Realizada la evaluación más exhaustiva se encontró una trombosis de seno venoso, la cual presenta una evolución hacia la mejoría con alta médica a los 15 días y recuperación de su estado de consciencia después de terapia anticoagulante. Los exámenes revelan una enfermedad protrombótica asociada. Se realizó revisión de la literatura y se establecieron los factores de riesgo asociados más frecuentes.

Palabras clave: trombosis de seno venoso, convulsiones posparto, enfermedades cerebrovasculares, eclampsia, síntomas neurológicos, factores de riesgo.

\section{SUMMARY}

The case of a young woman presenting neurological symptoms during post-natal care (puerperium) is described; she suffered from headaches and seizures after delivery in the obstetric ward. Differential diagnosis included postpartum eclampsia or infection.

* Presentado en el Congreso Colombiano de Perinatología. Medellín 2007. Póster.

** Residente de Ginecología y Obstetricia. Universidad del Valle. Hospital Universitario del Valle, Departamento de Ginecología y Obstetricia, Cali (Colombia). Correo electrónico: alherguz01@hotmail.com

*** Especialista Ginecología y Obstetricia. Universidad del Valle. MSc Obstetricia Universidad Autónoma de Barcelona. Profesor Asistente. Departamento de Ginecología y Obstetricia. Universidad del Valle, Cali (Colombia).
More exhaustive evaluation revealed cerebral venous sinus thrombosis (CVST); her state improved until hospital discharge after 15 days and recovery of her state of awareness following anticoagulant therapy. The exams revealed associated prothrombotic illness. The pertinent literature was reviewed and the most frequently associated risk factors and differential diagnoses were established.

Key words: venous sinus thrombosis, postpartum seizure, cerebrovascular disorder, eclampsia, neurological symptoms, risk factors.

\section{INTRODUCCIÓN}

La trombosis de seno venoso (TSV) es una condición caracterizada por la trombosis de las venas cerebrales y los senos mayores, que ocasionan edema cerebral, infarto venoso e hipertensión endocraneal. ${ }^{1-3}$ Ocurre en mujeres entre 20 y 25 años de edad, durante el embarazo (12\%), puerperio (60-80\%) y en usuarias de anticonceptivos orales, con un riesgo incrementado de 30\%. ${ }^{4}$ En $80 \%$ de las pacientes los factores de riesgo identificados abarcan: estados protrombóticos genéticos y adquiridos, infecciones, estados inflamatorios, hematológicos, traumáticos, cáncer y deshidratación. ${ }^{5}$

Asimismo, la trombosis de seno venoso se presenta con síntomas variables, donde la cefalea y las convulsiones son más frecuentes, por lo tanto, el diagnóstico se sospecha clínicamente y se confirma por resonancia magnética nuclear. ${ }^{6,7}$ 
El diagnóstico diferencial debe realizarse con la eclampsia posparto. ${ }^{8}$ Además, la TSV generalmente cursa con buen pronóstico, por lo que algunos casos pueden no ser diagnosticados; y entre 57 y $86 \%$ de los casos se logra una recuperación completa de la funcionalidad. ${ }^{9}$

Por consiguiente, el objetivo de la presentación del caso es mostrar la importancia del diagnóstico diferencial cuando una paciente en el puerperio desarrolla convulsiones. La consideración y la realización del diagnóstico de la TSV modifican la terapia y el pronóstico.

\section{CASO CLIÍNICO}

Paciente de 20 años de edad, sin antecedentes médico quirúrgicos importantes, que consultó al hospital de Caicedonia, Valle del Cauca, por presentar cefalea y estado post-ictal de episodio convulsivo tónico clónico generalizado; y pérdida súbita de la agudeza visual, con cianosis peribucal y evidencia de relajación de esfínteres, tensión arterial de 160/110 mmHg, y con antecedente de cesárea 4 días antes y diagnóstico de trabajo de parto estacionario. El recién nacido pesó: 2.800 g, Apgar: 9-10, con alta médica al día siguiente por presentar evolución satisfactoria. Se consideró el diagnóstico de eclampsia posparto y se procedió a realizar el tratamiento con dosis de sulfato de magnesio, 2 g de impregnación, 1 g/hora de mantenimiento. La paciente fue remitida al Hospital Universitario del Valle, Cali (Colombia), donde ingresó consciente, alerta, con escala Glasgow 15/15, presión arterial (PA) 143/83 mmHg, con frecuencia cardíaca (FC) 80 latidos/min, con frecuencia respiratoria (FR) 20 respiraciones/min, saturación de oxígeno de $98 \%$, útero sub-involucionado, cicatriz quirúrgica sana, loquios escasos fétidos, pupilas isocóricas normo reactivas, no papiledema, con agudeza visual de 20/800 en el ojo derecho y 20/200 en el ojo izquierdo, sin alteración motora ni sensitiva, hiperreflexia global, no clonus ni rigidez nucal.

Los exámenes iniciales indicaron: hemoglobina 10,2 mg/dL, lactato deshidrogenasa (LDH) $638 \mathrm{U} / \mathrm{L}$, fibrinógeno $630 \mathrm{mg} / \mathrm{dL}$, plaquetas normales, serología no reactiva. Por su parte, las pruebas de función hepática, función renal, coagulación y electrolitos séricos también estuvieron dentro de los límites normales.

La escanografía cerebral simple mostró múltiples imágenes hipodensas córtico-subcorticales bilaterales con lesión de múltiples territorios vasculares, parasagitales hacia la región parietal bilateral y occipital derecha, sugestivos de infartos venosos, edema cerebral difuso con borramientos de senos y cisternas y ventrículos laterales sin desviación de la línea media. En la resonancia magnética nuclear cerebral con gadolinio y la angiografía venosa se observaron imágenes hiperdensas en T1 y T2 que comprometen seno longitudinal superior y seno recto parcialmente e imágenes hipointensas en T1 e hiperintensas en T2 en cuerpo calloso, occipital y cerebelo sugestivo de infarto venosos por trombosis del seno longitudinal. Figura 1.

Los diagnósticos iniciales incluyeron eclampsia, trombosis de seno venoso y endometritis poscesárea, para lo cual se realizó tratamiento con sulfato de magnesio, nifedipina, clindamicina y gentamicina, medidas antiedema cerebral con manitol y solución salina hipertónica, anticoagulación formal con heparina no fraccionada en infusión continua y posteriormente manejo con warfarina, con la cual se logró anticoagulación con dosis alternantes de 5 y 7,5 mg/día.

En el $4^{\circ}$ día de manejo con heparina, la agudeza visual fue 20/50 bilateral y se ordenó seguimiento ambulatorio con oftalmología. Asimismo, se realizó monitoreo diario de la función renal con creatinina sérica, electrolitos, hemograma completo y tiempos de coagulación. Además, se llevó a cabo una escanografía simple cerebral al quinto día del ingreso en la cual se observó una mejoría del edema cerebral, sin evidencia de lesiones hemorrágicas. Igualmente, se practicaron anti-cardiolipinas IgG 7,9 GPL (negativo) y ANTICOAGULANTE LÚPICO (positivo).

Los diagnósticos definitivos fueron: trombosis del seno longitudinal superior, síndrome de anticuerpos antifosfolípidos y endometritis poscesárea 
Figura 1. RMN corte sagital y axial. Oclusión por trombosis del seno venoso e infartos cerebrales: occipital, cuerpo calloso y cerebelo.

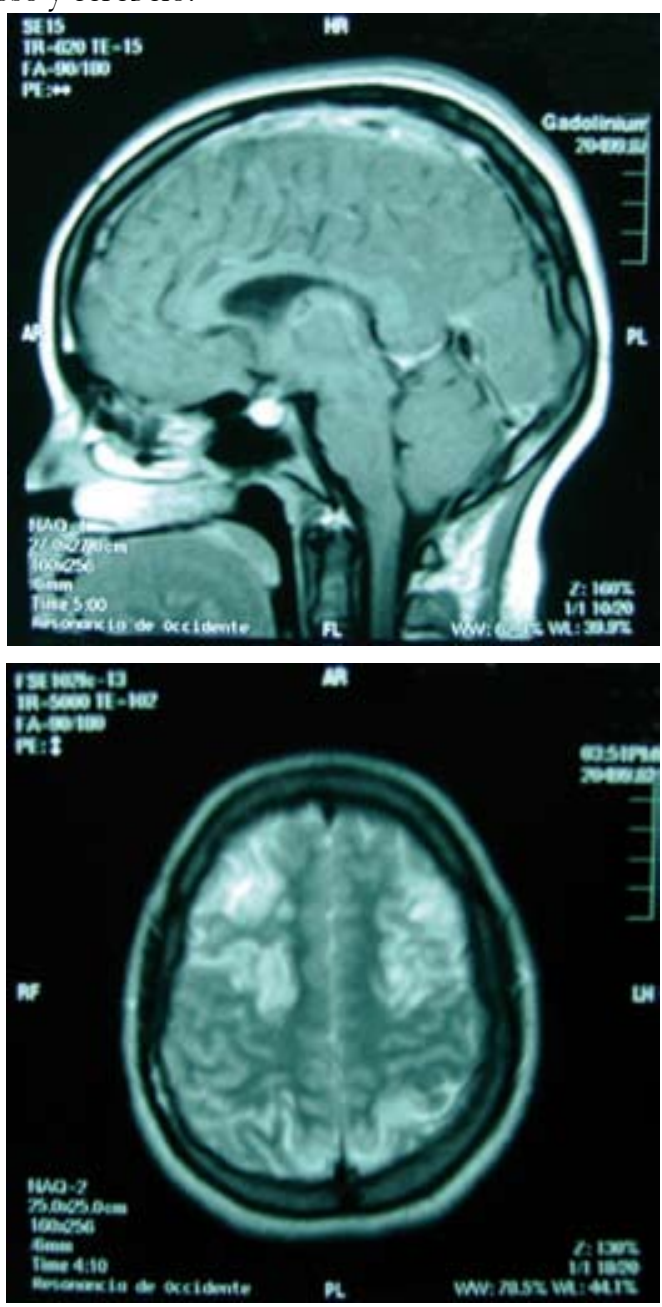

resuelta. Finalmente, la paciente fue dada de alta a los 12 días de estancia hospitalaria con warfarina y con controles ambulatorios por neurocirugía.

\section{DISCUSIÓN}

La trombosis cerebral de seno venoso (TSV) es una condición cambiante debido a su variabilidad de signos y síntomas clínicos y es muy frecuente no reconocerla en sus presentaciones clínicas iniciales. ${ }^{10}$ Ocurre en varios senos simultáneamente, y con mayor frecuencia, en el seno venoso transverso (86\%), seguido del seno sagital superior (69\%). ${ }^{11}$ Figura 1.

Los factores de riesgo corresponden a condiciones genéticas protrombóticas que incluyen: déficit de trombina, deficiencia de las proteínas $\mathrm{S}$ y $\mathrm{C}$, mutación del factor $\mathrm{V}$ de Leyden, homocisteinemia; estados protrombóticos adquiridos como: el síndrome nefrótico, anticuerpos antifosfolípidos, embarazo y puerperio; deshidratación y cáncer; e infecciones locales de cabeza, de cara, sistémicas, inflamatorias, hematológicas, traumáticas y por medicamentos. ${ }^{12}$ Tabla 1.

El diagnóstico, por su parte, se debe considerar en toda mujer que presente algún síntoma neurológico durante el embarazo y el puerperio y en todos los casos de hemorragia intracerebral de causa no explicada. Además, la TSV se manifiesta clínicamente con un amplio espectro de síntomas como lo son: cefalea en 70-90\% de los casos, convulsiones (47\% de los casos), déficit focal como hemiparesia (43\% de los casos), disturbios hemisensoriales, afasia, náuseas, vómito, alteraciones visuales, fotofobia, deterioro de la conciencia y papiledema en 25 a 33\% de los casos. El inicio puede ser agudo, subagudo o insidioso y prolongarse por días y semanas. ${ }^{46}$ En el presente reporte de caso, el síntoma principal en la paciente fue la cefalea, con posterior presentación de cuadro convulsivo y alteraciones visuales.

El diagnóstico se realiza por imagenología. El examen más sensible es la resonancia magnética nuclear $(\mathrm{RMN})$, combinada con la venografía por resonancia magnética, las cuales han desplazado a la escanografía y a la angiografía cerebral. ${ }^{13}$ En este caso, el diagnóstico fue realizado por RMN.

Por otro lado, la prioridad en el tratamiento es revertir el edema cerebral para evitar la herniación cerebral. La anticoagulación con heparina es el manejo necesario a fin de evitar nuevos eventos trombóticos y embolismo pulmonar que agravarán la condición del paciente. En 40\% de los casos se presentarán infartos hemorrágicos aún antes de iniciar la terapia anticoagulante. ${ }^{14}$ Una vez superada la fase aguda, debe iniciarse la anticoagulación oral con warfarina, durante 3 a 6 meses posparto, además de profilaxis y manejo terapéutico anticonvulsivante. Por el contrario, el tratamiento con trombólisis endovascular está limitado a centros con experiencia y con disponibilidad de radiología intervencionista. 


\section{Tabla 1. Causas y factores de riesgo asociados a trombosis de seno venoso}

\section{Condiciones genéticas protrombóticas}

Déficit de antitrombina

Deficiencia de proteínas $\mathrm{C}$ y $\mathrm{S}$

Mutación factor $\mathrm{V}$ de Leyden

Mutación de protrombina

Homocisteinemia

\section{Infecciones}

Otitis, sinusitis, mastoiditis

Meningitis

Infecciones sistémicas

\begin{tabular}{l} 
Hematológicas \\
\hline Policitemia \\
Trombocitopenia \\
Leucemia \\
Anemia \\
\hline \\
\end{tabular}

Anticonceptivos orales

Asparaginasa

\section{Estado protrombótico adquirido}

Síndrome nefrótico

Anticuerpo antifosfolípido

Homocisteinemia

Embarazo y puerperio

\section{Inflamatorias}

Lupus eritematoso sistémico

Granulomatosis de Wegener

Sarcoidosis

Síndrome de Behçet

\section{Traumáticas}

Injuria

Cateterización venosa

Neurocirugía

Punción lumbar

Deshidratación

Cáncer
En su mayoría, esta condición se desarrolla con buen pronóstico, ya que la mortalidad por esta causa ocurre entre 5,5 a $18 \%$ de los casos. De 57 a $86 \%$ de las pacientes recuperan totalmente la funcionalidad, y recurren en $12 \%$ a $14 \%$. Asimismo, en $57 \%$ de ellas no se manifiestan síntomas, 22\% de las pacientes presentan síntomas menores, y tan sólo 5\% síntomas mayores; mientras que $45 \%$ requieren manejo anticonvulsivante por largo tiempo. ${ }^{15}$

\section{AGRADECIMIENTOS}

Al doctor Carlos Hernández, médico residente de neurocirugía de la Universidad del Valle y al doctor Álvaro Caicedo, médico radiólogo del Hospital Universitario del Valle, por su colaboración en el diagnóstico y manejo de este caso.

\section{REFERENCIAS}

1. Stam J. Thrombosis of the cerebral veins and sinuses. N Engl J Med 2005;352:1791-8.
2. Allroggen $\mathrm{H}$, Abbott RJ. Cerebral venous sinus trombosis. Postgraduate Medical Journal 2000;76:12-15.

3. Kimber J. Cerebral venous sinus thrombosis. Q J Med 2002;95:137-142.

4. Karnad DR, Guntupalli KK. Neurologic disorders in pregnancy. Crit Care Med 2005;33:S362-71.

5. Ferro JM, Canhão P, Stam J, Bousser MG, Barinagarrementeria F; ISCVT Investigators. Prognosis of cerebral vein and dural sinus thrombosis: results of the International Study on Cerebral Vein and Dural Sinus Thrombosis (ISCVT). Stroke 2004;35:664-70.

6. Kittner S, Stern B, Feeser B, Hebel R, Nagey DA, Buchholz DW, et al. Pregnancy and the risk of stroke. N Engl J Med 1996;335:768-74.

7. Cole B, Criddle LM. A case of postpartum cerebral venous thrombosis. J Neurosci Nurs 2006;38:350-3.

8. Acheson J, Malik A. Cerebral venous sinus thrombosis presenting in the puerperium. Emerg Med J 2006;23:e44.

9. Atxer A, Pardina B, Blas I, Ramió L, Villalonga A. Dural sinus thrombosis in a late preeclamptic woman. Can J Anesth 2004;51:1050-1. 
10. FinkJN, McAuley DL. Cerebral venous sinus thrombosis: a diagnostic challenge. Intern Med J 2001;31:384-90.

11. Agid R, Shelef I, Scott J, Farb R. Imaging of the intracranial venous system. Neurologist 2008;14:12-22.

12. Lanska DJ, Kryscio RJ. Risk factors for peripartum and postpartum stroke and Intracranial venous thrombosis. Stroke 2000;31:274-82

13. Idbaih A, Boukobza M, Crassard I, Porcher R, Bousser MG, Chabriat H. MRI of clot in cerebral venous thrombosis. High diagnostic value of susceptibilityweighted Images. Stroke 2006;37;991-5.

14. Stam J, De Bruijn SF, DeVeber G. Anticoagulation for cerebral sinus thrombosis. Cochrane Database Syst Rev 2002:CD002005.

15. Mehraein S, Ortwein H, Busch M, Weih M, Einhäupl K, Masuhr F. Risk of recurrence of cerebral venous and sinus thrombosis during subsequent pregnancy and puerperium. J Neurol Neurosurg Psychiatry 2003;74:814-6.

Conflicto de intereses: ninguno declarado. 